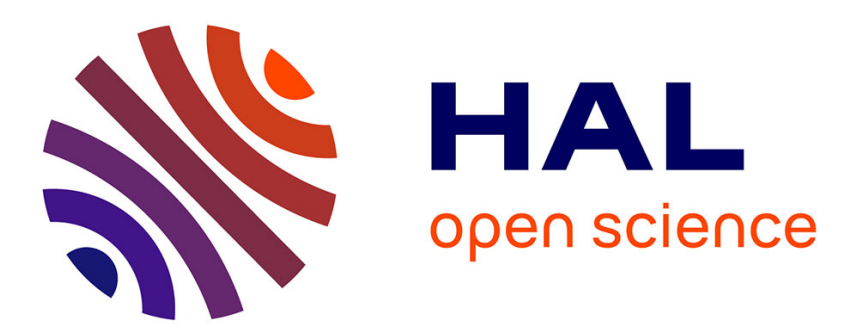

\title{
Deux lettres en une. Du Camp et Flaubert à Gautier, de Jérusalem, le 13 août 1850 \\ Sarga Moussa
}

\section{To cite this version:}

Sarga Moussa. Deux lettres en une. Du Camp et Flaubert à Gautier, de Jérusalem, le 13 août 1850. Bulletin de la Société de Théophile Gautier, 2020, Flaubert et Gautier, affinités électives, 42, pp.41-54. hal-03092161

\section{HAL Id: hal-03092161 \\ https://hal.science/hal-03092161}

Submitted on 1 Jan 2021

HAL is a multi-disciplinary open access archive for the deposit and dissemination of scientific research documents, whether they are published or not. The documents may come from teaching and research institutions in France or abroad, or from public or private research centers.
L'archive ouverte pluridisciplinaire HAL, est destinée au dépôt et à la diffusion de documents scientifiques de niveau recherche, publiés ou non, émanant des établissements d'enseignement et de recherche français ou étrangers, des laboratoires publics ou privés. 


\section{Deux lettres en une. Du Camp et Flaubert à Gautier, de Jérusalem, le 13 août 1850}

On connaît bien la correspondance d'Orient de Gustave Flaubert ${ }^{1}$, qui a souvent fait oublier celle de son compagnon de voyage, Maxime Du Camp. Plus généralement, il en est de même de l'œuvre de ces deux écrivains : l'un est passé à la postérité comme le précurseur de notre modernité littéraire,

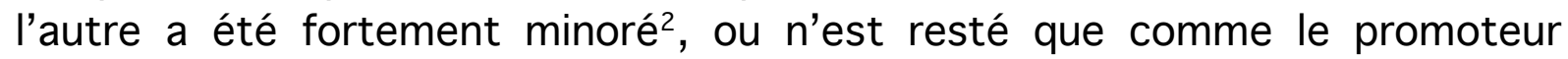
d'une littérature " utilitaire ", à l'opposé d'une conception intransitive que prônait déjà Théophile Gautier.

En réalité, lorsque Flaubert et Du Camp partent pour l'Orient, à la fin de l'année 1849, les jeux ne sont pas encore faits. Du Camp a déjà publié un récit de voyage (Souvenirs et paysages d'Orient, 1848), tandis que Flaubert a dû reconnaître l'échec de sa première version de la Tentation de saint Antoine, ayant accepté (bien qu'à contre-cœur) le jugement négatif de ses deux amis, Louis Bouilhet et Maxime Du Camp, à la suite de la lecture intégrale, à haute voix, du manuscrit.

II faut également rappeler qu'à cette époque, Du Camp n'est pas encore " saint-simonisé ${ }^{3}$ ", comme il le sera lorsqu'il commencera à publier ses premiers textes de fiction, et notamment son recueil poétique Les Chants modernes $(1855)^{4}$, où il célèbre les bienfaits de la modernité industrielle : ce sera un point de désaccord majeur avec Flaubert, dont il reste encore assez proche, notamment quant au refus de mêler littérature et politique, au moment où les deux compagnons accomplissent leur périple méditerranéen.

\footnotetext{
${ }^{1}$ La bibliographie est considérable sur ce sujet. On trouvera un certain nombre de titres d'ouvrages et d'articles sur ce sujet dans Éric Le Calvez (dir.), Flaubert voyageur, Paris, Garnier, 2019.

${ }^{2}$ Voir cependant le récent volume collectif Maxime Du Camp polygraphe, sous la direction de Thierry Poyet, La Revue des lettres modernes, 2019-4, Paris, Classiques Garnier, 2019. Antérieurement, il faut signaler les travaux de Marta Caraion, notamment Pour fixer la trace. Photographie, littérature et voyage au milieu du XIXe siècle, Genève, Droz, 2003, et les rééditions procurées par Daniel Oster, en particulier son édition, avec Michel Dewachter, du Nil (1854) de Maxime Du Camp (Un voyageur en Égypte vers 1850, Paris, Sand/Conti, 1987), ainsi que la biographie de Gérard de Senneville, Maxime Du Camp, un spectateur engagé du XIXe siècle, Paris, Stock, 1996.

${ }^{3}$ Sur cette question, voir Sarga Moussa, "Orient et saint-simonisme chez Maxime Du Camp. Des récits de voyage aux Mémoires d'un suicidé», dans Philippe Régnier (dir.), Études saint-simoniennes, Lyon, PUL, 2002, p. 245-270.

${ }^{4}$ Voir l'édition récente et le commentaire qu'en a procuré Marta Caraion dans Les Philosophes de la vapeur et des allumettes chimiques. Littérature, science et industrie en 1855, Genève, Droz, 2008.
} 
Gustave est l'aîné de Maxime de quelques mois, mais c'est ce dernier qui mène la barque : s'agissant des voyages, c'est lui qui est déjà l'homme d'expérience ; c'est donc lui qui convainc Mme Flaubert de laisser partir son fils ; et c'est lui qui obtient une véritable mission de photographe, lui aussi qui veut se faire rapidement un nom dans les lettres. Ce qui ne veut pas dire que Flaubert ne soit pas en train de méditer sur son avenir littéraire : il sait déjà qu'il veut consacrer toute sa vie à l'écriture, et, à ce titre, on peut lire parfois ses lettres et ses notes de voyage comme un laboratoire de l'œuvre à venir. Mais justement, autour de l'année 1850, Flaubert est dans la rétention littéraire, alors que Du Camp, dès son retour à Paris, fait feu de tout bois.

Cette asymétrie se retrouve dans la double lettre que Du Camp et Flaubert envoient à Gautier, de Jérusalem, le 13 août 1850. Au départ, il s'agit bien d'une lettre assez longue de Du Camp (trois pages dans la Correspondance générale de Théophile Gautier, c'est la lettre numérotée 1453). Mais Du Camp, avant de clore définitivement sa missive, laisse un espace (le verso du dernier feuillet), bien que significativement réduit, à Flaubert (" cette page blanche que Maxime me laisse ${ }^{5}$ ", écrit celui-ci). On va donc examiner, ici, tout à la fois ce qui unit et ce qui distingue les deux compagnons de voyage, leurs passions communes mais aussi leurs spécificités, thématiques ou stylistiques et, pour commencer, leur posture respective face à leur destinataire, Théophile Gautier, resté à Paris.

\section{Le rôle de Gautier}

Du Camp, dans ses Souvenirs littéraires (1882-1883), écrit avoir connu Gautier "par l'intermédiaire de Louis de Cormenin ", dans les années qui suivirent la Révolution de $1848^{6}$. À l'intérieur de la correspondance de Flaubert, il apparaît pour la première fois dans une lettre à sa mère, datée de Paris, le 28 octobre 1849 : "Bouilhet est arrivé ce matin à 11 h[eures]. Nous dînons tous les trois ensemble avec Théophile Gautier, qui a remis une invitation pour venir avec nous ${ }^{7}$. " Ces quelques mots sont déjà riches d'enseignement. Flaubert part en Orient avec Du Camp, mais en réalité, ils auraient pu former un trio : Bouilhet sera le grand absent, celui auquel Flaubert se confiera sans retenue, dans de très longues lettres, comme pour faire participer, par procuration, l'ami absent aux jouissances de ce voyage. Autre indication révélatrice : Gautier, de dix ans leur aîné, a " remis » une invitation pour pouvoir dîner avec ce petit club d'hommes de lettres en

\footnotetext{
5 Théophile Gautier, Correspondance générale, sous la resp. de Pierre Laubriet, éd. Claudine Lacoste-Veysseyre, Genève-Paris, Droz, t. IV (1849-1851), 1989, p. 208.

${ }^{6}$ Maxime Du Camp, Souvenirs littéraires, éd. Daniel Oster, Paris, Aubier, 1994, p. 283.

7 Gustave Flaubert, Correspondance, t. I, éd. Jean Bruneau, Paris, Gallimard, «Bibliothèque de la Pléiade », 1973, p. 517.
} 
devenir8. Autrement dit, l'homme déjà bien installé qu'est Gautier, à la fois poète, prosateur, critique d'art et journaliste, n'a pas hésité à annuler un rendez-vous pour ne pas manquer le départ de la jeune garde littéraire prête à accomplir le rituel périple méditerranéen - celui-là même que Gautier, d'ailleurs, ne pourra jamais faire entièrement : il lui faudra attendre l'été 1852 pour pouvoir séjourner à Constantinople et en Grèce, puis 1869 pour connaître l'Égypte, pourtant déjà utilisée comme matière romanesque. Le " grand " Gautier, donc, parraine symboliquement ses cadets, mais il les envie aussi, puisque c'est eux qui s'apprêtent à réaliser le rêve oriental ${ }^{9}$.

Voici comment Du Camp commence la double lettre qu'il envoie avec Flaubert à Gautier, de Jérusalem, le 13 août 1850 :

Le dernier courrier venu de France et qui nous est arrivé avant-hier ici m'a apporté, mon cher Fortunio, dans une lettre de Louis de Cormenin, les mots du souvenir à notre adresse qui terminaient votre feuilleton du 24 juin $^{10}$.

L'allusion à la grande nouvelle de Gautier (celle qu'il considérait lui-même comme sa dernière œuvre écrite en totale liberté, sans la contrainte quotidienne du journalisme) sera reprise en conclusion de la lettre de Du Camp ("Adieu, mon cher Fortunio ${ }^{11}$ ") : c'est ici comme écrivain que Gautier est considéré, et, du même coup, c'est une complicité littéraire, tournée vers l'Orient, que Du Camp recherche en identifiant le nouvelliste à son héros indien. L'un des enjeux du voyage est donc, pour lui, de devenir écrivain.

Notons par ailleurs que cette lettre commence par une allusion à un feuilleton, celui que Gautier avait publié dans le journal La Presse du 24 juin

\footnotetext{
${ }^{8}$ Dans ses notes de voyage, Flaubert précise: "Visite à Gautier que nous invitons à dîner » (Voyage en Orient, dans Gustave Flaubert, OEuvres complètes, t. II [1845-1851], sous la dir. de Claudine Gothot-Mersch, Paris, Gallimard, "Bibliothèque de la Pléiade », 2013, p. 596).

${ }^{9}$ Sa réponse à la double lettre que Du Camp et Flaubert lui enverront est d'ailleurs tout à fait claire à ce sujet, de même qu'y apparaît nettement le statut supérieur du premier par rapport au second, puisque Gautier répond ainsi (oubliant Flaubert) : « Mon cher Maxime, que puis-je vous écrire, sinon que je suis bassement jaloux de votre bonheur et que j'envie le sort de votre domestique? Je claque d'ennui et je me donne des coups de pied au cul toute la journée pour ma lâcheté. Je voudrais voler la Banque de France, assassiner quelque bourgeois, suriner [= frapper] un capitaliste et vous aller rejoindre [...] ». Lettre de Théophile Gautier à Maxime Du Camp, dans Gautier, Correspondance générale, t. IV, éd. citée, p. 273 (lettre 1499). La date proposée, celle du 13 décembre 1850 , semble peu probable, puisqu'il s'agit bien d'une réponse à la lettre de Du Camp et Flaubert datée du 13 août 1850 à Jérusalem - elle est plus probablement du mois de septembre de la même année.
}

10 Lettre de Du Camp à Gautier, dans Gautier, Correspondance générale, t. IV, éd. citée, p. 205.

11 Ibid., p. 207. 
1850, et qui se terminait par une sorte de message personnalisé à Du Camp, message qui se voulait lui-même une réponse à une lettre envoyée précédemment par ce dernier. Gautier avait donc écrit ces mots, dans une sorte de mini-lettre ouverte où il adoubait par avance un Maxime Du Camp aux talents multiples :

\begin{abstract}
Au moment d'achever notre feuilleton, nous recevons une lettre des pays les plus extravagants, - une lettre mise à la poste à la seconde cataracte du Nil ! Elle est signée "Maxime Ducamp ", un intrépide voyageur de nos amis, qui avec son camarade Flaubert, est en train de photographier des palais de Pharaon, d'estamper des bas-reliefs, de prendre des notes, par une température de four à plâtre, sur les rives du gand fleuve où, la nuit, les sphinx altérés de la chaleur du jour, descendent laper l'eau jaune de leur langue de granit rose. Cette lettre, imprégnée de soleil, chaude et cuite de ton comme un Marilhat ou un Guignet, pleine de science, d'esprit et de cordialité, demande une réponse que nous irions bien volontiers porter nous-même à l'auteur de ce beau voyage en Orient [les Souvenirs et paysages d'Orient] dont Pelletan a rendu compte dans La Presse, mais nous sommes retenu ici par la patte, et là où le feuilletoniste est attaché, il faut qu'il fasse l'analyse des vaudevilles $[\ldots]^{12}$.
\end{abstract}

Gautier participe donc à la promotion de l'identité sociale multiple que Du Camp est en train de se fabriquer pour lui-même : photographe orientaliste, voire archéologue amateur, mais aussi et surtout écrivain du voyage, l'expression " voyageur intrépide » permettant de réunir la double image, savante et littéraire, que le jeune homme s'emploie à construire et à donner de lui-même.

Quant à la lettre de Du Camp à laquelle Gautier fait allusion, elle nous a été conservée, et elle commençait déjà par la formule "mon cher Fortunio ", un nom qui est d'ailleurs repris deux fois, d'abord d'une manière volontairement désinvolte, sur un ton familier qui dénote la complicité que l'épistolier cherche à créer avec son destinataire, promoteur de " l'art pour l'art » (« [...] je vous soupçonne d'une paresse épistolaire si grande que je n'attends rien d'un Fortunio comme vous »), puis, en conclusion de cette même lettre, sous la forme d'une sorte de mini-résumé de ce que pouvait symboliser le héros de cette nouvelle, incarnation d'un Orient source de toute richesse (« Quant à vous, cher Fortunio, je vous souhaite bonne muse, plein d'or dans les poches et bonheur sempiternel $\gg)^{13}$ - c'est là un quasi-pastiche de la préface de Gautier à sa grande nouvelle ${ }^{14}$

\footnotetext{
12 Ibid., p. 208-209, n. 1.

13 Lettre $n^{\circ} 1377$, de Du Camp à Gautier, «En Nubie, descendant le Nil, à la hauteur de Teskra », 31 mars 1850, dans Gautier, Correspondance générale, t. IV, éd. citée, p. 134.

14 «Fortunio est un hymne à la beauté, à la richesse, au bonheur, les trois seules divinités que nous reconnaissions » (Théophile Gautier, Fortunio et autres nouvelles, éd. Anne Bouchard, Lausanne, L'Âge d'homme, 1977, p. 23).
} 
Mais revenons à la double lettre de Du Camp et de Flaubert. Le premier y développe longuement l'histoire de deux obélisques de Louxor, I'un ayant été transporté en France pour être érigé sur la place de la Concorde, l'autre étant resté en Haute-Égypte - occasion pour Du Camp de construire une histoire sur le thème de l'éternelle insatisfaction identitaire, sujet dont il souffle à Gautier tout à la fois la matière et l'idée qu'il pourrait lui dédier ce poème lorsqu'il le rédigera ${ }^{15}$ : c'est exactement ce que fera ce dernier avec le grand poème "Nostalgies d'obélisques ${ }^{16}$ ". L'Orient est donc, pour Gautier, comme, avant lui, pour Hugo et pour Goethe, littéralement, source de poésie ${ }^{17}$.

Quant à Flaubert, il se place dans une posture un peu plus modeste que Du Camp vis-à-vis de Gautier, tout en se permettant lui aussi une attitude relevant de la familiarité affectueuse : " Je vous embrasse, cher maître tout le long de cette page blanche que Maxime me laisse. Quittez donc Paris, volez n'importe qui ou n'importe quoi, - si les fonds sont bas -, et venez avec nous. Quel soleil ! quel ciel ! quels terrains, quel tout ! Si vous saviez !18 » Attitude anti-bourgeoise, voire anarchiste et subversive, prônée dans une forme de complicité dont on verra tout à l'heure quelques-unes des composantes - mais Flaubert en reste néanmoins au " cher maître ", dans la posture respectueuse de celui qui sait qu'il a encore tout à prouver en ce qui concerne ses talents littéraires, et qui est conscient qu'il doit en passer par une forme d'adoubement par celui dont le nom peut ouvrir des portes - il continuera d'ailleurs, par habitude, à employer cette formule dans la plupart des lettres qu'il écrira à Gautier, jusqu'à la fin de la vie de ce dernier.

\section{La fuite du temps}

Dans ces deux lettres qui se suivent, Du Camp et Flaubert expriment à Gautier des préoccupations communes, qui sont liées, notamment, à la hantise du temps qui passe. On peut supposer avec une certaine

\footnotetext{
15 « [...] j'ai vu là un bon sujet de vers, une bonne matière, comme nous disions au collège et je vous l'envoie. Si vous la faites, dédiez-là moi » (lettre du 13 août 1850, dans Gautier, Correspondance générale, t. IV, éd. citée, p. 206 ; souligné dans le texte).

${ }^{16}$ C'est dans le première publication de « Nostalgies d'obélisques » (La Presse, 4 août 1851) que figure la dédicace à Du Camp, laquelle disparaît de la reprise du poème dans Émaux et Camées (1852) - voir l'édition de ce recueil procurée par Claudine GothotMersch, Paris, Gallimard, «Poésie», 1981, p. 60-65 et les notes correspondantes, p. 244 et suiv., sur la dette de Gautier vis-à-vis de Du Camp.

17 Voir Sarga Moussa et Michel Murat (dir.), Poésie et orientalisme, Paris, Classiques Garnier, 2019.

18 Nous citons d'après la Correspondance de Flaubert procurée par Jean Bruneau, t. I, éd. citée, p. 663 (souligné par l'auteur), le texte figurant à la suite de la lettre de Du Camp et donné dans la Correspondance générale de Gautier (t. IV, éd. citée, p. 208) étant moins exact.
} 
vraisemblance que Du Camp et Flaubert ont lu, chacun, le contenu de la lettre de l'autre, ce qui, soit dit en passant, relativise la confidentialité sur laquelle est censée reposer la pratique épistolaire. Quoi qu'il en soit, il y a incontestablement une circulation de l'information, qui fait des deux destinateurs et du destinataire une sorte de trio dont la complicité repose sur des valeurs communes. L'une d'elles est le sentiment que, comme le dit Du Camp en citant Musset, "le christianisme a fait son temps ${ }^{19}$ ". Arriver à Jérusalem, au milieu du XIX siècle, n'a pas la même signification que pour un voyageur comme Chateaubriand, ou même comme Lamartine. Le premier faisait de la Ville sainte le but de son Itinéraire (1811), conçu comme la réactivation d'un pèlerinage vers les sources de la civilisation chrétienne de la France. Le second, dont la foi était déjà ébranlée au moment où il partait pour l'Orient, présentera néanmoins la visite du Saint-Sépulcre comme un grand moment de son Voyage en Orient (1835). On entre, avec les années 1840-1850, dans une crise religieuse qui se transforme, parfois, en " ère du soupçon " avant la lettre. La chose est même sensible chez la comtesse de Gasparin qui, au nom de la "vérité de la foi ${ }^{20}$ ", reconnaît : " Je ne sais comment cela se fait, il y a sans doute de ma faute ; mais je n'ai pu rencontrer encore à Jérusalem cette solitude avec mon Sauveur que j'espérais ${ }^{21}$. " Gasparin est une protestante genevoise, et en ce sens, sa critique vise évidemment les dogmes de la religion catholique. Mais elle formule aussi, prudemment, une autocritique ( il y a sans doute de ma faute »), qui laisse entendre qu'il devient difficile d'atteindre à la plénitude attendue lorsqu'on visite Jérusalem, où se presse une multitude de pèlerins croyant marcher dans les pas du Christ, et où coexistent plusieurs cultes chrétiens à l'intérieur même du Saint-Sépulcre, dont les clés sont par ailleurs entre les mains du gouverneur ottoman de la ville.

Du Camp, de son côté, franchit un pas de plus : pour lui - et on verra qu'il en est presque de même pour Flaubert -, le Dieu des juifs et des chrétiens est mort, et l'évocation des différentes étapes de la Palestine parcourues sur les traces des anciens pèlerins tourne à la parodie provocatrice :

La majeure partie de la population est composée de Juifs qui viennent mourir ici de tous les coins du monde afin d'aller pourrir en compagnie de leurs pères dans la vallée de Josaphat - [...] - tout cela est singulièrement grotesque et je voudrais bien savoir sous quelle religion vivront les enfants illégitimes de nos fils naturels. Demain au soleil levant, nous partons avec une respectable escorte de gredins en guenilles, pour visiter Jéricho, le Jourdain et la mer Morte ; là, je rechercherai pour la plus grande joie de ces bons pédérastes l'emplacement

${ }^{19}$ Dans Gautier, Correspondance générale, t. IV, éd. citée, p. 207.

20 [Valérie de Gasparin], Journal d'un voyage au Levant (1848), 2e éd., Paris, Ducloux et Cie, 1850, t. III, p. 161.

${ }^{21}$ Ibid., p. 170. 
positif de Sodome, cette respectable cité que Dieu a détruite par une impardonnable et puérile susceptibilité de pudibonderie $[\ldots]^{22}$.

Faut-il voir, outre la provocation, une dimension antisémite, au sens moderne du terme, dans les propos de Du Camp ? C'est bien probable. Et l'honnêteté oblige de dire que Gautier n'y était sans doute pas insensible, preuve en est une page de Constantinople (1853) où Balata, le quartier juif de la capitale ottomane, est décrit dans les termes les plus abjects ${ }^{23}$. Quant à Flaubert, dans la lettre qu'il écrit à la suite de celle de Du Camp, il ne verse pas dans ce travers raciste et ne s'exprime pas sur la mort de Dieu, mais il parodie lui aussi la tradition des pèlerins cherchant à retrouver le site des villes pécheresses en se rendant à la mer Morte, allant jusqu'à suggérer (est-ce par provocation ?) une tentation homosexuelle ${ }^{24}$. En revanche, dans une autre lettre qu'il écrit de Jérusalem, cette fois-ci en son nom seul, on perçoit une dimension nostalgique dans l'évocation d'une Ville sainte dont la présence divine s'est absentée. II s'agit d'une longue missive adressée à Louis Bouilhet :

Je n'ai pas pleuré sur ma sécheresse ni rien regretté, mais j'ai éprouvé ce sentiment étrange que deux hommes comme nous éprouvent lorsqu'ils sont tout seuls au coin de leur feu et que, creusant de toutes leurs forces de leur âme ce vieux gouffre représenté par le mot amour, ils se figurent ce que ce serait... si c'était possible. Non, je n'ai été là ni voltairien, ni méphistophélique, ni sadiste. J'étais au contraire très simple. J'y allais de bonne foi, et mon imagination même n'a pas été remuée ${ }^{25}$.

II y a donc un même sentiment, chez Du Camp et Flaubert, de ne pas retrouver dans la Jérusalem moderne les traces de l'histoire biblique. Mais on perçoit chez Flaubert une déception absente chez son compagnon, et qui est due, sans doute, au souvenir douloureux de la lecture de la première version de Saint Antoine, juste avant le départ : ce que son auteur attendait secrètement de la ville sainte, peut-être, c'était quelque chose comme une confirmation de son choix littéraire, celui d'écrire un grand ouvrage sur les tout débuts du christianisme - mais justement, ce qui intéresse Flaubert, à travers le personnage de l'anachorète traversé de visions hétérodoxes, ce sont en réalité les dieux, ceux d'une époque où la Vérité unique, celle du

\footnotetext{
${ }^{22}$ Lettre de Du Camp à Flaubert, de Jérusalem, dans Gautier, Correspondance générale, t. IV, éd. citée, p. 207.

${ }^{23}$ Voir Théophile Gautier, Constantinople et autres textes sur la Turquie, éd. Sarga Moussa, Paris, La Boîte à Documents, 1990, p. 210-211.

24 «Demain matin au soleil levant nous partons pour Jericho et la [mer] Morte. Nous allons donc voir la place où fut Sodome - quelle idées ça va fai[re] naître en nous !? » (Flaubert, Correspondance, t. I, éd. citée, p. 664).

${ }^{25}$ Lettre de Flaubert à Bouilhet, de Jérusalem, le 20 août 1850, dans Correspondance, t. I, éd. citée, p. 666-667 ; souligné dans le texte.
} 
christianisme triomphant, ne s'était pas encore imposée. Quant à Gautier, il partageait avec les deux rédacteurs de la double missive qu'il recevait de Jérusalem (où il ne s'est d'ailleurs jamais rendu) un même point de vue critique à l'égard du Dieu chrétie ${ }^{26}$; des œuvres comme Fortunio et le Roman de la momie témoignent en tout cas d'un intérêt certain pour le paganisme.

La deuxième grande hantise liée à la fuite du temps que Du Camp et Flaubert partagent avec Gautier, est celle de l'européanisation de l'Orient. Chez le premier, c'est moins dans la lettre de Jérusalem à Gautier, que dans une lettre antérieure au même, envoyée par Du Camp depuis la Nubie, que cette dimension apparaît : " [...] dépêchez-vous [de venir en Égypte], le temps presse : ici on démolit les temples pour en faire des fabriques à sucre $[\ldots]^{27}$. " C'est la politique de réformes menée par les vice-rois depuis Mohammed Ali que vise Du Camp - une politique qui a parfois menacé le patrimoine pharaonique, ce dont Champollion s'inquiétait déjà lors de son propre voyage en Égypte, à la fin des années $1820^{28}$. Or, on retrouve la même inquiétude concernant une modernité envahissante, mais à propos d'autres objets, dans la lettre que Flaubert ajoute à celle de Du Camp, depuis Jérusalem : " II est temps de se dépêcher. D'ici à peu l'Orient n'existera plus. Nous sommes peut-être des derniers contemplateurs. - Vous ne vous doutez pas de tout ce qui est déjà sali ; les soldats turcs ont des souspieds ! J'ai vu passer des harems dans des bateaux à vapeur ${ }^{29}$. " C'est là un grand thème chez les voyageurs du milieu du XIX siècle, celui de la critique des tanzimat, les réformes à l'européenne qu'on associe en particulier à l'ère du sultan Abdul-Medjid (1839-1861) ${ }^{30}$. Toute forme d'hybridité culturelle, dès lors qu'une des composantes en serait d'origine occidentale, est en général condamnée par les voyageurs français dans l'empire ottoman au milieu du XIX siècle $^{31}$. L'Orient dont Du Camp, Flaubert et Gautier sont en

${ }^{26}$ Voir Philippe Berthier, "L'injure chrétienne», dans «Panorama Gautier », dossier dirigé par Sarga Moussa et Paolo Tortonese, Revue des Sciences humaines, $\mathrm{n}^{\circ} 277,2005$, p. 15-25.

27 Lettre de Du Camp à Gautier, « En Nubie, descendant le Nil, à la hauteur de Teskra », le 31 mars 1850, dans Gautier, Correspondance générale, t. IV, éd. citée, p. 133.

${ }^{28}$ Voir la Note remise au vice-roi pour la conservation des monuments de l'Égypte, datée d'Alexandrie, novembre 1829, et reproduite par Markus Messling dans Les Hiéroglyphes de Champollion. Philologie et conquête du monde, trad. fr. par Kaja Antonowicz, Grenoble, ELLUG, «Vers l'Orient », 2015, p. 117 et suiv.

${ }^{29}$ Flaubert, Correspondance, t. I, éd. citée, p. 663. Même tonalité parodique dans une lettre à Bouilhet datée du lazaret du Pirée, à Athènes, le 19 décembre 1850 : «Ô Orient, où es-tu ? - Il ne sera bientôt plus que dans le soleil. À Constantinople, la plupart des hommes sont habillés à l'européenne, on y joue l'opéra, il y a des cabinets de lecture, des modistes, etc ! » (ibid., p. 730).

30 Voir Robert Mantran (dir.), Histoire de l'empire ottoman, Paris, Fayard, 1989.

31 Voir les actes du colloque qui s'est tenu au lycée Notre-Dame-de-Sion (Istanbul) les 15 et 16 mars 2019, «Écrivains, musiciens et artistes face aux tanzimat. Enjeux esthétiques 
quête est un Orient fantasmé comme autre. L'idée d'un impérialisme civilisateur leur est totalement étrangère - ce qui ne veut pas dire qu'ils sont sans préjugé « orientaliste », comme on va le voir.

\section{L'hyper-sexualité}

Le discours sur le christianisme comme religion du passé (surtout chez Du Camp), auquel s'ajoute la critique de l'européaanisation de l'empire ottoman (plutôt chez Flaubert), a pour contrepartie implicite une valorisation de l'islam, ou plus exactement un imaginaire de l'islam, rêvé, en l'occurrence, comme la possibilité d'une sexualité démultipliée autorisée par la polygamie. Or, Du Camp et Flaubert partagent, à cette époque-là, tout à la fois un ensemble de pratiques sexuelles et une même liberté de ton quant à la façon d'en parler (qui va de la grivoiserie à la provocation), ce dont ils ne se privent pas dans leur lettre respective à Gautier - ils connaissent également le côté " noceur » de celui-ci, qui peut aussi se traduire par une certaine crudité de langage dans sa correspondance ${ }^{32}$.

Mais revenons à la lettre que Du Camp envoie à Gautier de Jérusalem :

À Luxor, ô Théo, j'ai beaucoup pensé à vous et voici pourquoi - Parce qu'il y a un obélisque - cela peut vous sembler étrange, car dans vos bottes ou sous votre chapeau, vous n'avez qu'une ressemblance fort éloignée avec ces incommodes godmichés [sic] que les Égyptiens plaçaient en érection devant les portes béantes de leurs temples - et cependant c'est l'obélisque qui m'a fait songer à vous ${ }^{33}$.

Le terme de godemiché (phallus artificiel) renvoie vraisemblablement à Sade, référence commune aux trois amis ${ }^{34}$. Notons au passage l'apostrophe « Ô Théo ", qui joue plaisamment sur le sens du prénom de Gautier (Théophile = qui aime Dieu), de façon à faire du plaisir sexuel non pas un objet de culpabilité, comme dans la tradition chrétienne, mais au contraire une activité

et idéologiques », sous la dir. de Nicolas Dufetel et Sarga Moussa, à paraître à Istanbul aux éditions Isis.

32 «Jamais trop de cul - [...] - jamais trop de métaphores ! voilà ma devise » (lettre de Gautier à Flaubert, 28 novembre 1856) ; voir Gautier, Correspondance générale, t. VI, éd. Claudine Lacoste-Veysseyre, Genève, Droz, 1995, p. 236 - lettre figurant également (avec une datation exacte) dans l'édition établie par Yvan Leclerc et Danielle Girard de l'édition électronique de la Correspondance de Flaubert: URL: https://flaubert.univrouen.fr/jet/public/correspondance/feuilletage.php?t=D\&sens=T\&c=GAUT.

${ }^{33}$ Lettre de Du Camp à Gautier, de Jérusalem, le 13 août 1850, dans Gautier, Correspondance générale, t. IV, éd. citée, p. 205.

34 Voir la lettre de Flaubert à Gautier datée de Croisset, 30 mai 1857 : « Je t'attends. Je m'arrangerai pour procurer à mes hôtes un De Sade complet! Il y en aura des volumes sur les tables de nuit !» (Correspondance, t. I, éd. citée, p. 727 ; souligné par Flaubert). 
à laquelle il s'agit de rendre un culte. Depuis Jérusalem, on parlera donc non pas de Jésus, mais de sexe.

Le genre épistolaire, dans la mesure où il est censé rester dans le domaine privé, peut offrir un espace de liberté que ne comporterait pas un texte publié. La lettre se situe ici dans le cadre d'une circulation restreinte entre un groupe de personnes qui partagent une certaine complicité. Ainsi Du Camp poursuit-il sa lettre à Gautier par la mention de sa rencontre, à Beyrouth, d'un ami de ce dernier, Camille Rogier, dont on sait qu'il procura aux deux compagnons de voyage des prostituées"35. "Quoi que nous ne [nous] fussions jamais aperçus, nous nous sommes vite reconnus de la même famille », écrit Du Camp - et la suite du texte est tout à fait explicite quant au sens qu'il faut accorder à cette « famille »:

\footnotetext{
J'ai daigné concourir, chez lui, à l'accroissement de la race musulmane en m'y superposant à quatre ou cinq drôlesses qui avaient des vêtements de velours, des pantalons de soie, des mains teintes de henné, des yeux bordés d'antimoine et des boisseaux de piastres d'or dans leurs cheveux - quelques jours après j'ai été obligé de me mettre sous la protection du dieu Mercure, divinité bienfaisante qui vous guérit des maux de Vénus - grâce à son influence, ce détail est passé $[\ldots]^{36}$.
}

Comme dans l'extrait précédemment commenté, ce qui est frappant, ici, c'est que le " tableau de chasse ", que Du Camp amplifie sans doute à plaisir, comme le fait Flaubert au même moment dans ses lettres à Bouilhet, va de pair avec une certaine créativité linguistique, qui permet d'allier des considérations plus ou moins scabreuses à un savoir mythologique partagé à quoi s'ajoutent des allusions médicales (le mercure servait à cicatriser les chancres : Flaubert, lui aussi fils de médecin, y fera également allusion dans sa correspondance d'Orient ${ }^{37}$ ). L'Orient féminisé et sexualisé est ici bien proche, il faut le reconnaître, de l'« orientalisme » dominateur stigmatisé par Edward Said ${ }^{38}$

\footnotetext{
${ }^{35}$ Le peintre et dessinateur Camille Rogier (1810-1896) était directeur des postes à Beyrouth. Flaubert l'évoque à plusieurs reprises dans sa correspondance, en particulier dans une lettre à Bouilhet où il dit qu'il est « de la clique Gautier » (de Jérusalem, 20 août 1850, ibid., p. 668).

${ }^{36}$ Lettre de Du Camp à Gautier, dans Gautier, Correspondance générale, $\mathrm{t}$. IV, éd. citée., p. 206 ; le «nous » ajouté entre crochets figure dans le manuscrit (Bibliothèque de l'Institut, coll. Lovenjoul, C 493, $\mathrm{f}^{\circ} 433$ ).

37 «Il faut que tu saches, mon cher monsieur, que j'ai gobé à Beyrouth (je m'en suis aperçu à Rhodes, patrie du dragon) VII chancres, lequels ont fini par se réunir en deux, puis en un » (lettre à Bouilhet, de Constantinople, le 14 novembre 1850, dans Flaubert, Correspondance, t. I, éd. citée, p. 707).

38 Voir Edward W. Said, Orientalism (1978), trad. fr. par Catherine Malamoud, L'Orientalisme. L'Orient créé par l'Occident, Paris, Éditions du Seuil, 1980.
} 
On observera qu'il y a dans l'extrait de cette lettre de Du Camp une forme d'exotisme qui cherche à séduire jusque dans le registre sexuel le maître descripteur qu'était Gautier, comme s'il fallait racheter une certaine brutalité de l'acte sexuel répété par un processus d'esthétisation féminine (vêtements, tatouage, maquillage, accessoires des prostituées). En comparaison, le même épisode, raconté du point de vue de Flaubert, est décrit avec beaucoup plus de crudité, dans un style " réaliste " qui laisse place à des détails démythifiants : "Elle avait le nez un peu retroussé et de la chassie au bord de la paupière intérieure de l'œil droit 39 ", écrit-il à propos de l'une des trois femmes avec lesquelles il dit avoir couché avant le déjeuner. On mettra ce regard microscopique hallucinant de précision ${ }^{40}$ en relation avec la façon dont Flaubert avait décrit Kuchuk-Hanem, la prostituée de Haute-Égypte dont il a tout à la fois célébré la beauté plantureuse ( " narines fendues - larges épaules solides - seins abondants, pomme ${ }^{41}$ ») et consigné dans ses notes cette observation impitoyable : " elle a une incisive d'en haut, côté droit, qui commence à se gâter ${ }^{42}$. " Flaubert écrira plus tard, à Louise Colet : "Tu me dis que les punaises de Kuchiouk-Hânem te la dégradent ; c'est là, moi, ce qui m'enchantait » - et de généraliser sous la forme d'une esthétique à venir : " Je veux qu'il y ait une amertume à tout, un éternel coup de sifflet au milieu de nos triomphes, et que la désolation même soit dans l'enthousiasme ${ }^{43}$. "

Le voyage en Orient, pour Flaubert comme pour Du Camp, a parfois quelque chose d'un laboratoire de l'œuvre à venir, et à ce titre, la correspondance et les notes de voyage révèlent peu à peu des préférences esthétiques. Gautier est comme l'arbitre de ces galops d'essai, en tout cas le témoin qui les reçoit de manière tout à la fois bienveillante et complice. Les grivoiseries elles-mêmes traduisent des orientations stylistiques. Brodant sur le thème de l'érotisation pharaonique (l'obélisque en forme de godemiché), Du Camp va plutôt du côté du symbolisme, tandis que Flaubert, lui, penche vers un réalisme qui peut d'ailleurs virer parfois au grotesque (« Au Caire, j'ai vu un singe masturber un âne. L'âne se débattait, le singe grinçait des dents,

${ }^{39}$ Lettre à Bouilhet, de Jérusalem, le 20 août 1850, dans Flaubert, Correspondance, t. I, éd. citée, p. 668.

${ }^{40}$ Voir à ce sujet l'article de Nathalie Petibon, «Flaubert en Italie. De la mort du rêve à la naissance du style », dans Éric Le Calvez (dir.), Flaubert voyageur, op. cit., p. 237-249, en particulier p. 247 pour l'attention extrême aux détails les plus infimes, notamment dans les visages décrits par Flaubert.

${ }^{41}$ Gustave Flaubert, Voyage en Orient, dans OEuvres complètes, t. II, éd. Claudine GothotMersch, Paris, Gallimard, «Bibliothèque de la Pléiade », 2013, p. 660.

42 Ibid.

43 Lettre à Louise Colet de Croisset, 27 mars 1853, dans Gustave Flaubert, Correspondance, t. II, éd. Jean Bruneau, Paris, Gallimard, «Bibliothèque de la Pléiade », 1980, p. 283. 
la foule regardait, c'était fort ", écrit-il à la fin de sa lettre de Jérusalem à Gautier $\left.^{44}\right)$.

$\star \star \star$

Nous avons ici un cas d'écriture à quatre mains - mais peut-être faudrait-il inclure aussi Gautier, comme " horizon d'attente »? -, écriture plurielle qui fragilise la notion d'auteur au sens classique du terme, et qui oblige à penser l'auctorialité comme un phénomène moins stable qu'il n'y paraît.

Deux modalités du récit de voyage émergent de cette comparaison. Pour Du Camp, l'Orient est d'abord l'occasion d'une épopée personnelle, c'est une aventure exotique dont il s'agit de montrer qu'il a la maîtrise : "Nous y sommes arrivés [à Jérusalem] venant de Beyrouth, après avoir suivi le rivage de la Phénicie pendant neuf jours, à cheval, paisiblement, sous un grand soleil, dormant à la belle étoile sous les caroubiers et les lauriers roses, sans souci des puces qui se nourrissaient de nous et repartant au point du jour précédés de nos Bédouins armés ${ }^{45}$. " Du Camp se pense comme un homme d'action nomade, il le dira encore dans ses Souvenirs littéraires, où il fait son autoportrait en contraste complet avec son compagnon de voyage, peint comme un introverti ${ }^{46}$. Du Camp n'a pas totalement tort, mais il faut tout de suite ajouter que le côté rêveur de Flaubert ne l'a pas empêché d'être un excellent observateur. II suffit de reprendre le passage de sa lettre de Jérusalem où il décrit à son tour le trajet au bord de la Méditerranée à pour s'en convaincre : " De Beyrouth à Jaffa il y a des bois de lauriers roses poussés tout au bord de la mer"4. " Flaubert ne narre pas cette étape, il décrit le paysage (" il y a ») dans son mode d'être, ou parfois dans son surgissement, ainsi que le révèle la belle image qu'il donne, toujours dans la même lettre, des chameaux " mélancoliques », qu'on voit " dans le désert s'avancer devant vous alignés sur un seul rang ${ }^{48} »-$ dans l'un et l'autre cas, le sujet de l'écriture tend à s'absenter de celle-ci : on pense, bien sûr, à la future esthétique flaubertienne de l'impersonnalité.

Dans leur double lettre de Jérusalem du 13 août 1850, Flaubert et Du Camp transforment le pèlerinage traditionnel en une quête littéraire dont leur maître et ami est le destinataire privilégié. Tout en partageant une même hantise de la modernité et un discours provocateur sur la sexualité, chacun

\footnotetext{
${ }^{44}$ Flaubert, Correspondance, t. I, éd. citée, p. 664.

45 Lettre de Du Camp à Gautier, de Jérusalem, le 13 août 1850, dans Gautier, Correspondance générale, t. IV, éd. citée, p. 205.

46 « Gustave Flaubert n'avait rien de mon exaltation, il était calme et vivait en lui-même. Le mouvement, l'action lui étaient antipathiques» (Maxime Du Camp, Souvenirs littéraires, éd. citée, p. 314).

47 Flaubert, Correspondance, t. I, éd. citée, p. 664.

48 Ibid., p. 663-664.
} 
développe une forme d'écriture qui lui est propre, mais qui peut trouver des échos chez Gautier lui-même - que ce soit dans son œuvre viatique ou poétique.

Sarga MOUSSA, CNRS, UMR THALIM (CNRS-Université Sorbonne Nouvelle-ENS) 